\title{
Robotics in clothes manufacture
}

\author{
R. C. Michelini, R. P. Razzoli" \\ University of Genova, DIME, Genova, Italy
}

Email address:

michelini@dimec.unige.it (R. C. Michelini), razzoli@dimec.unige.it (R. P. Razzoli)

To cite this article:

R. C. Michelini, R. P. Razzoli. Robotics in Clothes Manufacture. International Journal of Mechanical Engineering and Applications, Vol. 1, No. 1, 2013, pp. 17-27. doi: 10.11648/j.ijmea.20130101.13

\begin{abstract}
Process automation and intelligent manufacturing are important factors for competing successfully in today's international economy. The survey considers the use of these tools within the clothing industry where low cost labour has especially driven the marketplace, in recent decades, toward productive break-up, with relevant drawbacks on preserving the process control uniformity. Several hints are described for the use of modern manufacturing tools in the production flow of clothing and in the management of the related operations. Emphasis is placed on maintaining a high quality product in a customised production environment. The presentation begins by exploring the automated retrieval of fabric from a warehouse; it continues with a study of the laying and cutting process exploring mixed mode schedules, combining batch and one-of-a-kind delivery; and concludes by considering the assembly and sewing aspects of the manufacturing process. For clothing manufacturers in developed countries, the automation concepts presented here offer an alternative (and perhaps the only alternative) to out-sourcing and overseas production.
\end{abstract}

Keywords: Robotics in Textiles; Customised Clothes; Integrated Automation; Materials vs. Information Flows

\section{Introduction}

Robot concepts have loose applications in clothing manufacture, where the flow of information (from ideation and strategic planning, to shop programming, flexibility decisions and quality assessments) is distinct from the flow of material (from fabrics and supplies, to laying, cutting, assembly and sewing of suits, dresses and apparel). The manufacturers prefer manual work-cycles, moving labour intensive tasks to low-wage countries, with material flow fragmentation and overseeing discontinuity. Alternatively, the return on investment shall aim at using newer technologies to permit customer-driven product mixes within a quick-response time frame by controlled highest quality and reliability. Instead, creation of high quality clothing is viewed as handicraft; innovation limits at assisting skilled people at the ideation phases, with only concern in enhancing product value creation, from the customer's viewpoint. The Fig. 1 summarises a few features of the clothing industry related to intelligent manufacture: a number of options matches between the business and the capabilities of robotics. The Fig. 2 indicates the full spectrum of robot options, considering time scales, from operation ranges, to strategic planning.

Customer satisfaction is highly dependent
on changeable fashion-driven trends.
Quality features of clothing products
(unlike high-tech products) are readily understood.
Labour intensive material processing jobs
cover a broad range of skills.
Business skill is important for creating
new opportunities, but commonly does not
require new process technologies.
Quality monitoring, quick response, adaptive
scheduling, process visibility, etc. are basic opportuni-
ties.
Lean engineering, flexible automation,
total quality, piece-wise improvement result
fromeconomy of scope.
Heterogeneous (fashion-driving) details
do not affect fit-for-use properties.
Trade-marks (or brands) can be uniformly
used in world-wide advertising.
In-process monitoring algorithms are needed
to provide consistency with flexible,
adaptive manufacturing.

Figure 1. Integrated intelligence features in robot manufacture of clothing

Developed countries are interested in preserving quality-of-life, creating jobs and resources for the benefit of 
value-generating businesses, having products and processes compatible with the environment. From this viewpoint, the up grading of traditional manufacturing areas, such as textile and apparel, is very important; therefore, exploring approaches based on distributed intelligence is natural including concepts previously described in the literature $[1,2$, $3,4,5,6]$. Changes must be viewed in terms of expected returns and with the understanding that in many cases a near revolution must be accomplished to win back world-wide competitiveness $[7,8]$.

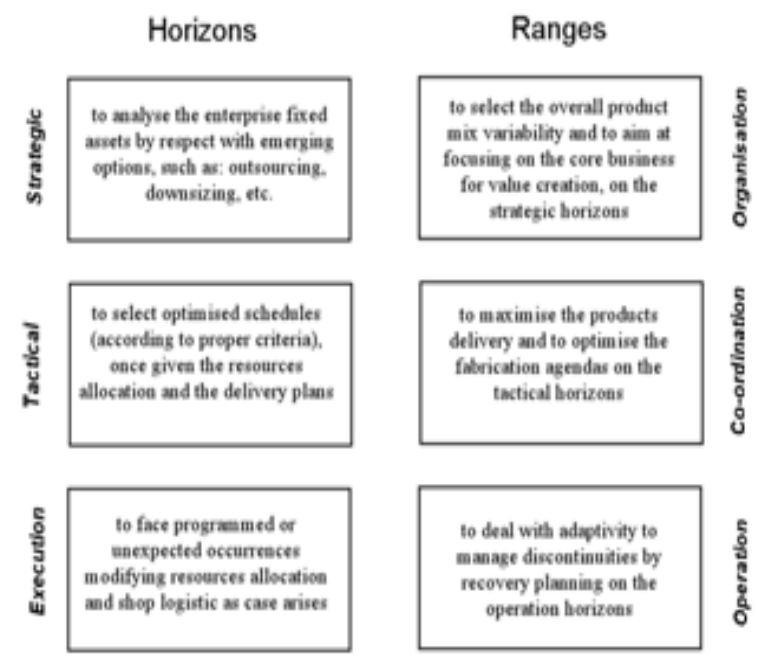

Figure 2. The full spectrum of manufacturing flexibility.

The manufacture of high-quality clothing is an area that benefits from advanced design concepts and from intelligent manufacturing technology. Items are sold with high added value, driven by consumer's satisfaction; marketing is global in scope with rapidly changing fashion trends. Buyers are right away aware of quality, reliability and value/price ratio; manufacturers are confronted by demanding challenges, with pressure on prices, new fashions, customisation, wide product mix, quick response with dependable delivery dates, etc. Economies of scope can replace economies of scale, following typical patterns, including flexible specialisation, lean engineering, company-wide quality, and continuous product improvement. Indeed, the creation of high-quality clothing includes skilled labour mainly at the ideation phases, and the strategy of keeping critical jobs inside the main factory and distributing outside labour intensive cycles might be pursued for prompt returns. On mid-term to long term horizons, a rather different strategy needs be considered, based on a series of steps, say:

- work-cycles and production schedules should balance on the strategic horizon, with entire exploitation of available resources;

- maintenance monitoring of the manufacturing process shall be carefully performed, aiming at steady quality, with zero-defect production;

- integrated control-and-management of plant versatility ought to be explored, delivering the product mix with just-in-time schedules, at the clients' due dates;
- product-and-process up grading need be piece-wise carried out, with transparent assessment of each intermediate achievement.

This steps fixed, certain manufacturers with a reputation for (and perhaps a focus on) quality start to seriously consider the technology to improve efficiency, aiming at enhancing the design of customised products, with attention on information flow, material procurement, marketing orders, etc., to promote innovative ideas managing process intelligence. However, automation in the manufacture of clothing presents some unique problems compared to other industrial areas, due to rather dissimilar motivations:

- the comparatively straightforward processing without dangerous tasks;

- the wide range of skills used in work-cycles, based on combined (rather than elemental) jobs;

- the substantial commitment required for the training of expert workers;

- the limp behaviour of fabrics and wide range of materials used during processing;

- the creation of 3D shapes by the warping of 2D pieces;

- and so forth.

The dependence of textile and clothing industries on automation, thus, requires explicit areas-adapted statements. There is no doubt that the mechanisation with ingenious rigs and automata developed in these areas (looms with task-driven commands, lace and embroidery needle-working machines, etc.), since front-end contrivances provided mass-production by off-line setting. However, recently, flexible manufacture concepts did remain at the outboard, with little innovation at front-end automation and low data integration by process and product monitoring.

The choice deserves notice, as it represents a peculiarity compared to extant trends that moves apparel business to the developing countries, with tricky outcomes, e.g., the habit of looking for short-use thrown-away items, without concern on quality and durability. Alternatively, changes aim at robot technologies; the approach explores the information flow criticality, when efficiently merged into the base material flow $[9,10]$.

The presentation recalls a series of projects especially concerned by high quality garments, where the value chain offered by the industrial organisation will out-perform handicraft production for safety and reliability. For explanatory purposes, three characterising manufacture sections are specially addressed:

the storage and the retrieval processes of fabric from the warehouse in preparation for manufacture of varying mixes of garments, as for type, model, size, etc.: efficiency depends on exploiting existing information, notably, on on-line managing the fabric quality-data, and this is enhanced by addition of purposely developed front-end handling equipment.

- the laying and cutting processes of garment components, today, already accomplished by programmable machines, i.e., noteworthy fixed automation rigs: attention is on intelligence for on-line adaptive flexibility; an example is 
considered to show productivity effects, when one-of-a-kind and batch products are simultaneously processed.

- the assembly and sewing processes of garments, mainly deferred to hybrid stands, with front-end operators interfaced to automation heads: scope (vs. scale) economy organisation is analysed and a benchmarking strategy allows evaluating the shop effectiveness by on-process managing the distributed intelligence.

The relevant role is played by the enterprise information systems and related data acquisition, storing and retrieval modules. These topics are today properly appreciated; the developed hardware and software tools are established technology and little needs be added to explore their transfer to textile and clothing industries $[11,12]$. The investigation is therefore more specifically interested to the tools supporting knowledge build-up and decision making, in direct connection with the considered business. Computer simulation is introduced as important aid for setting adaptive govern rules on the automation of the manufacturing process. We shall distinguish several types of aids. In a general way, resort to digital mock-ups and to virtual reality testing is fundamental engineering means to accomplish lifecycle tests on (digital) prototypes, so that the actual behaviour of possible (material) fixtures is properly assessed under the full scenarios of alternative forecast engagements.

Computer simulation is, thus, off-process aid at the design phase, to find out the most effective lay-out for given engineering specifications. Intelligent automation, moreover, confronts with varying duty environments, and conflicts with the concept of optimal setting. On-process computer simulation, then, is current decision support to up-date resource allocation and to adapt fabrication agendas according to economy of scope rules, with account of time horizons and planning ranges, Fig. 2.

The three example developments cover the different situations as for front-end automation. They, quite properly, represent reference cases, when the attention is focused on return on investment in material processing facilities. The present study reconsiders the problems widening the focus on the joint material-and-information flows management. The situation modifies, at least, when quality clothes are considered, where the value chain will naturally rise in terms of customised quality reliability. The up-grading does not face steady outcomes: at times, enhanced material flow automation is winning step to help data flow integration; sometimes, the automation level is already fully developed, and integration is straightforward; some other times, return on investment for further automation is questionable, and data flow integration could look after the inclusion of front-end workers. All in all, the developments provide a general view of the issues offered by distributed intelligence in the shop-floor organisation, when high quality garments manufacture is considered.

\section{Fabric Warehousing and Handling}

At first, the intelligent factory addresses «mater ials-and- information> integrated flow, so that combined inputs transform in deliveries with optimised value. The fabric supply is critical. The job shall provide the pertinent data on: warehouse inventory, cloth types and lengths, procurement policy, etc., for inventory and purchasing information. For production plans, special data for the stored rolls are required, including handling rules, characteristic features, fault maps and classification, etc., Fig. 3.

\begin{tabular}{|} 
Composition - Number of ends and picks - Weave \\
Colour fastness to light, to perspiration, to dry cleaning, to washing, to \\
dry pressing/ironing, to rubbing, to dry spotting, etc. \\
Breaking strength and elongation (longitudinal/transversal) - Tear en- \\
durance - Breaking strength (grab method) \\
Resistance to abrasion, to pilling, to snagging, to dry ravelling, etc. \\
Seam slippage - Crease recovery - Dimensional stability to washing, to \\
dry cleaning, etc. \\
Yarn defects: broken threats, curling, twin top yarn, uneven glossiness, \\
knots, etc. \\
Warp defects: barred weaving, furrows, stack, faulty warping, \\
chopped-off warp, etc. \\
Woof defects: lacking yarn, wrong weft, stretched/winding thread, fake \\
reach, etc. \\
Flaws after dying, printing, finishing; unevenness, stains, blurs, creases, \\
slobbers, etc. \\
Selvedge flaws: folded listing, holed selvage, damaged boundaries, etc. \\
Extra deficiencies: skew slant, drawing asymmetry, holes, inclusions, \\
uneven width, etc.
\end{tabular}

Figure 3. Typical features/defects of woven fabrics.

These data provide the background knowledge for front-end operations. They also provide foreground information, to implement combined control and management logistics.

In the manufacture of quality clothing, two guidelines for a successful return on investment are basically considered:

- for ready-made clothing, production efficiency is main request: the market demand for a given season should match the total number of suits or dresses prepared for that season; this would imply successful productivity decisions, made over a tactical time horizon;

- market-driven manufacturing should be available for quick response opportunities, so that customised or personalised items can be efficiently managed in process, within an operational time frame [13].

Fabric warehousing and retrieval technology is fair contribution in both cases. In ready-made production, efficiency and lean manufacturing concepts play a major role in scrap reduction and production effectiveness. In terms of flexible manufacturing for custom made clothing, the retrieval process is especially important to allow varying items mix or limited production runs to be inserted as case arises, without 
relevant downgrading the normal production schedule.

The Fig. 4 shows the basic elements of the fabric warehousing, exemplifying the roll storing frame, the pick/place rig. This has a suited gripper, Fig. 5, with twin sliding arms, carried by a mobile tower. This way, each single bolt has fully automatized handling and management.
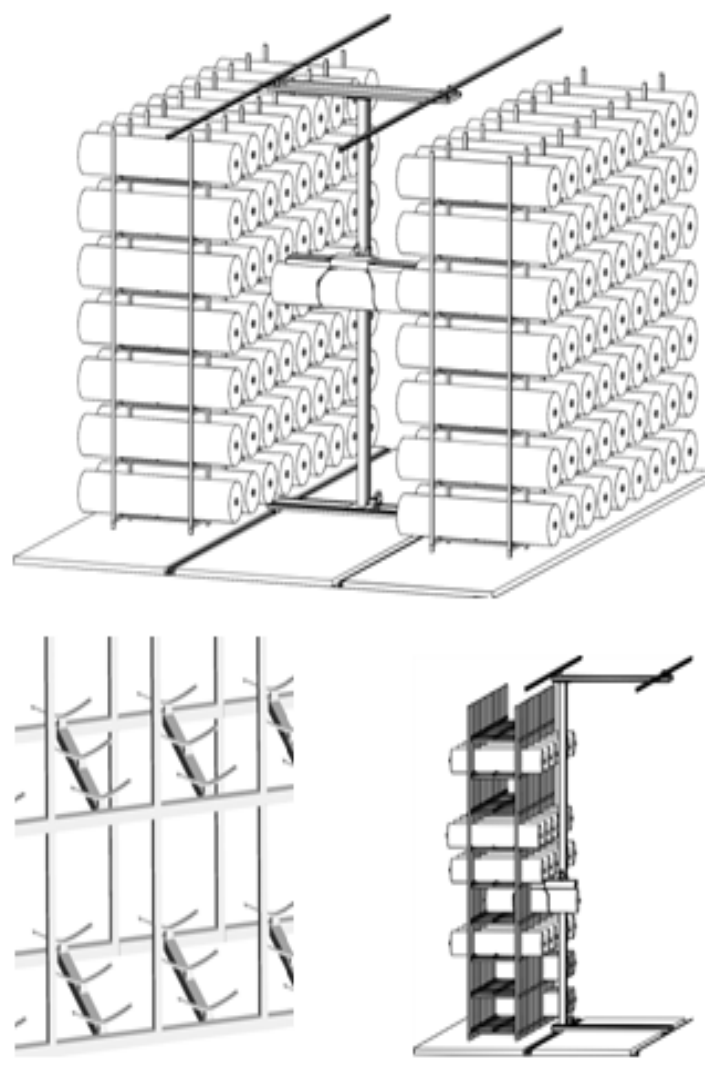

Figure 4. Fabric warehouse: layout, supporting frame, pick/place rig.
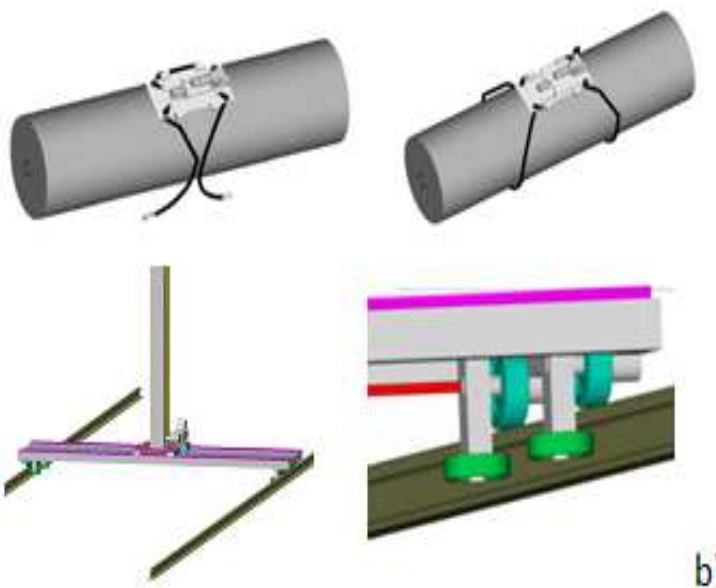

a)

b)

Figure 5. Roll grasping: engagement/latching; tower translation and roller details.

The computer-aided planning has recourse to a process simulation code, Fig. 6, describing the fabric flow, according to each especially detailed service. The controller performs the scheduling and coordination, minimising the waste and supplying lying and cutting data for further processing.

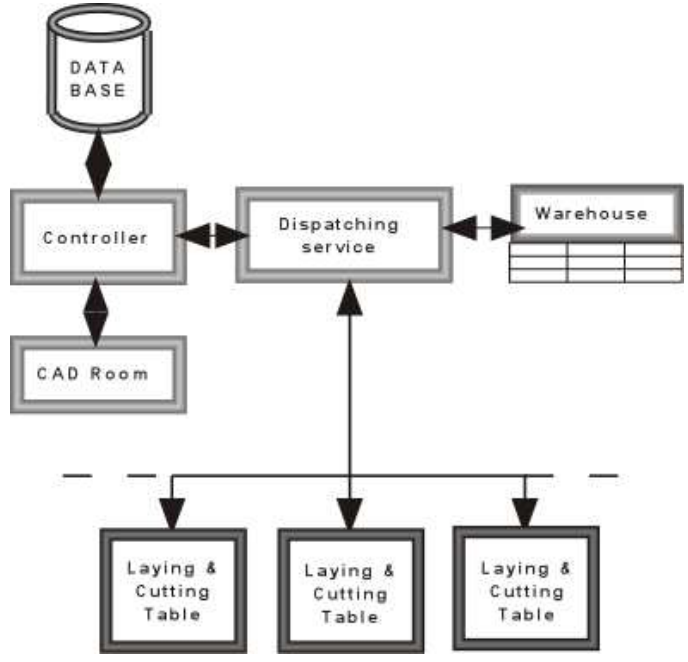

Figure 6. Architecture of the RSS-SIFIP simulation code.

The simulation code supplies off-process anticipation of the planned schedules, to test the actual achievements. Later, it permits, Fig. 7, viewing the individual production course, to verify the nested lay-out, with the pertinent details (e.g., location of defects, if any).

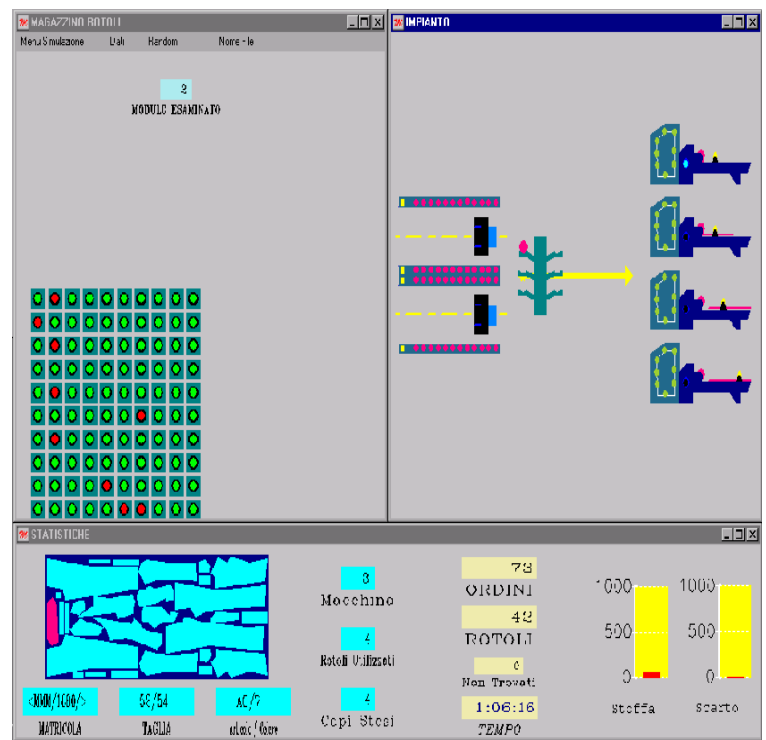

Figure 7. Example RSS-SIFIP window for the process monitoring.

The duty monitoring provides real-time data and statistical records. For example purpose, we consider the opportunity of mixing together batch orders and one-of-a-kind items (including replacements for flawed garment parts, as just mentioned). During on-line operations, the part layout choice and knowledge of the defect maps suggest special-nesting ideas to grant clothing quality or, alternatively, a subset of garment parts could result with excessive faults.

These appear marked-up on the display and replacement pieces would be separately cut from an extra length of the same fabric, Fig. 8 . 

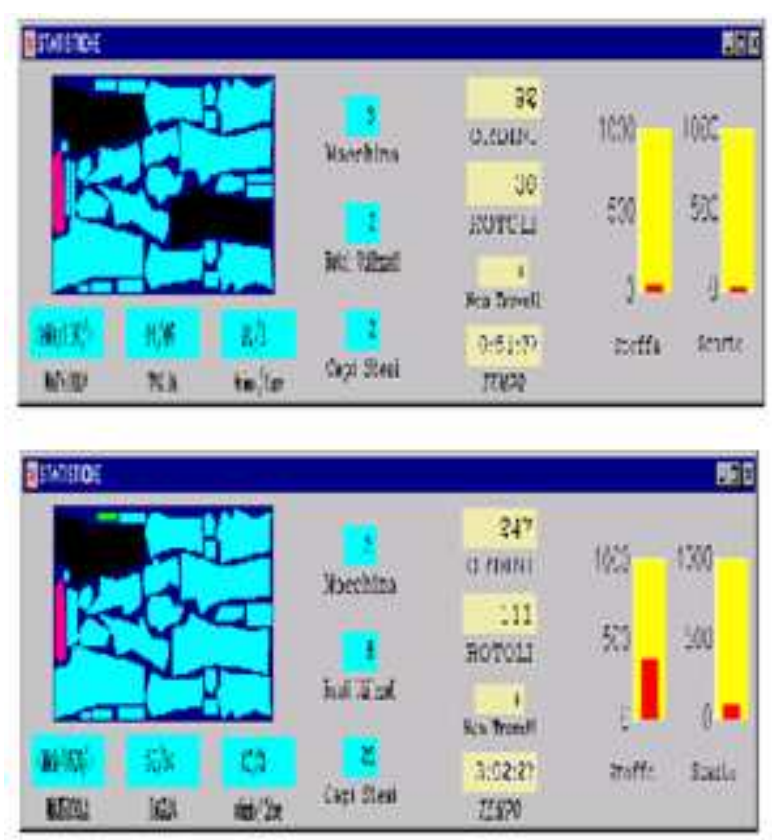

Figure 8. Windows for the management of defective parts.

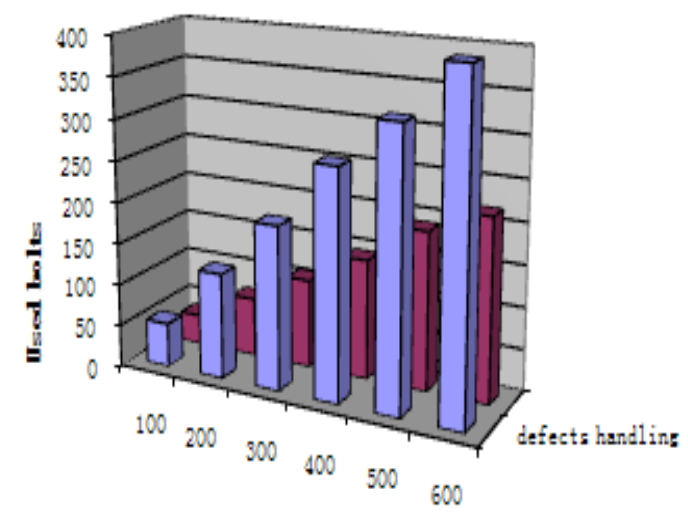

Nuber of orders

Figure 9. Handled bolts in case of on-process management of defected parts.

The simultaneous management of one-of-a-kind items is possible, on condition that the laying tables accept free spans for out-of-batch articles. This way, it easy to include extra items out of the original production plans straight in real time, or (e.g. when the desired fabric is temporarily not available), pushing the changes to a later batch. The adaptive planning adjustments, needed to allow such flexibility, can be provided by interaction with on-line operators or by an expert module, endowed of rule-based decision logic.

The example case data are: the warehouse section consists of four scaffolds with 10x10 frames, 400 total bolts and 20 equally distributed fabric types; the initial roll length ranges from 18 to $20 \mathrm{~m}$, with (duly mapped) 5 to 15 significant faults; the mix of garments requires a set number of cutting spans on the layout table. Depending on the batch size for the garments, the fabric laying process considering de- fect-mapping shows a considerable waste reduction, Fig. 8, compared to the fit post-cutting recognition of non-usable pieces. The in-process use of fabric data needs the handling of a larger number of bolts, Fig. 9, but this is balanced by the fact that later handling (of the same bolts) for cutting replacement pieces is avoided, also, removing any time-delay in the item delivery. In this case, the optimal bolt search turns to be simple due to a large number of choices.

The area of high-standing garments, satisfying varying market requests, is exemplary case where automation provides critical support for quality certification. A complex innovation of, both, material and information resources shall be promoted, to include:

- flexible/versatile cloth warehouse, with effective data-base storing and management capabilities;

- shop logistic service for fabric housing and dispatching, by on-process intelligent decision logic.

These changes need to be investigated in terms of the expected returns. Simulation studies are dominant help, to compare competing alternatives referring to actual production contexts and to provide explanatory checks with training purport related to sets of feasible implementations.

\section{Fabric Laying and Cutting}

The automation of the warehousing and retrieval process is logical start to establish a distributed intelligence organisation. The laying and cutting are already done by fully automatic stands, with long tables allowing the unwrapping of several superposed layers, where a sequence of 'windows' distinguish size or model of identical parts assortments. Productivity depends on the number of layers aimed at mass production. With customised products, the window accepts a single layer; the effect of the changes in product mix affects the strategic decision to automate (to deal with throughput and due-time wavers, by optimal top-down schedules); in lieu, the operation decision aims at customised garments (to face bottom-up requests, still preserving the structured frames of industrial production).

The fashion industry mainly operates on a seasonal basis with months of lead-time in view of each season. The manufacturers try to anticipate the tastes and whims of consumers and they attempt to stock sufficient quantities of garments, to meet the expected demand. Of course, this may not be economical for both under and over estimating demand has a cost. The flexible automation offers the potential for handling market driven orders on a more real-time basis, as well as providing improvements in efficiency in the production of customised garments. The joint batch and one-of-a-kind processing shall cover the typical sequence, Fig. 10:

- cloth bolts warehousing and selective materials delivery;

- fabric laying and cutting with pieces buffering and sorting;

- sewing sections with feeding and material logistics;

- pressing sections and items final finishing;

- quality checking, packing and garments storing; 
- supervision for retail supply and marketing.

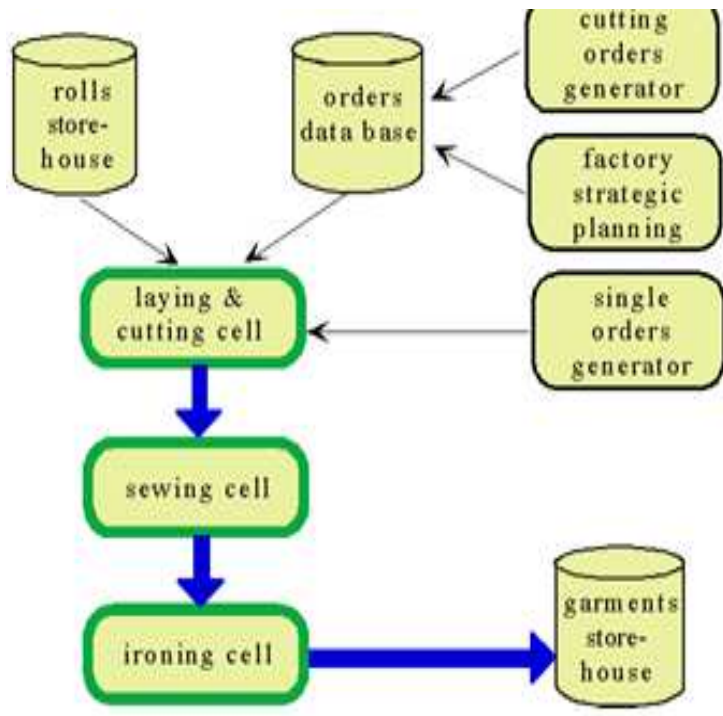

Figure 10. Information flow-chart of the garment manufacture process. ing cells. The production flow needs be analysed and the functional description coded for computer simulation.

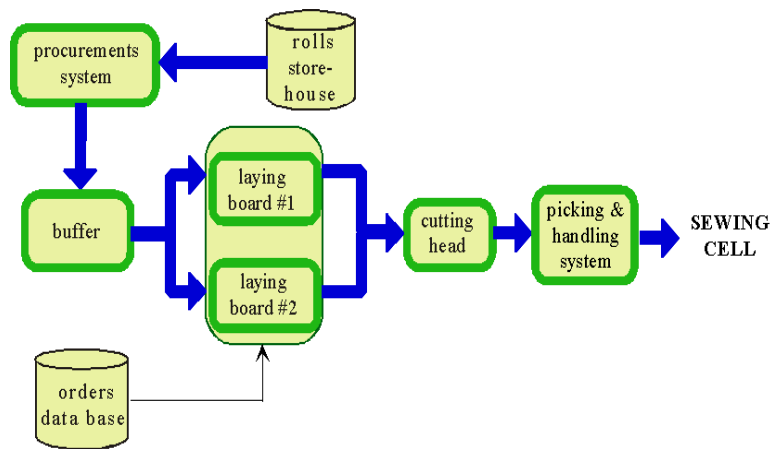

Figure 11. Architecture of the LCC-SIFIP simulation code.

The code utilises, Fig. 12, of two tables for unwrapping, alternatively feeding a cutting station. The display is surrounded by updated information on the production flow and on the utilisation ratio of the allotted resources.

The laying/cutting section is key step, to exploit. The facility, Fig. 11, receives information from the order data-base for the nested cutting, obtains fabric from the warehouse through a buffer, and delivers cut garment parts to the sew-

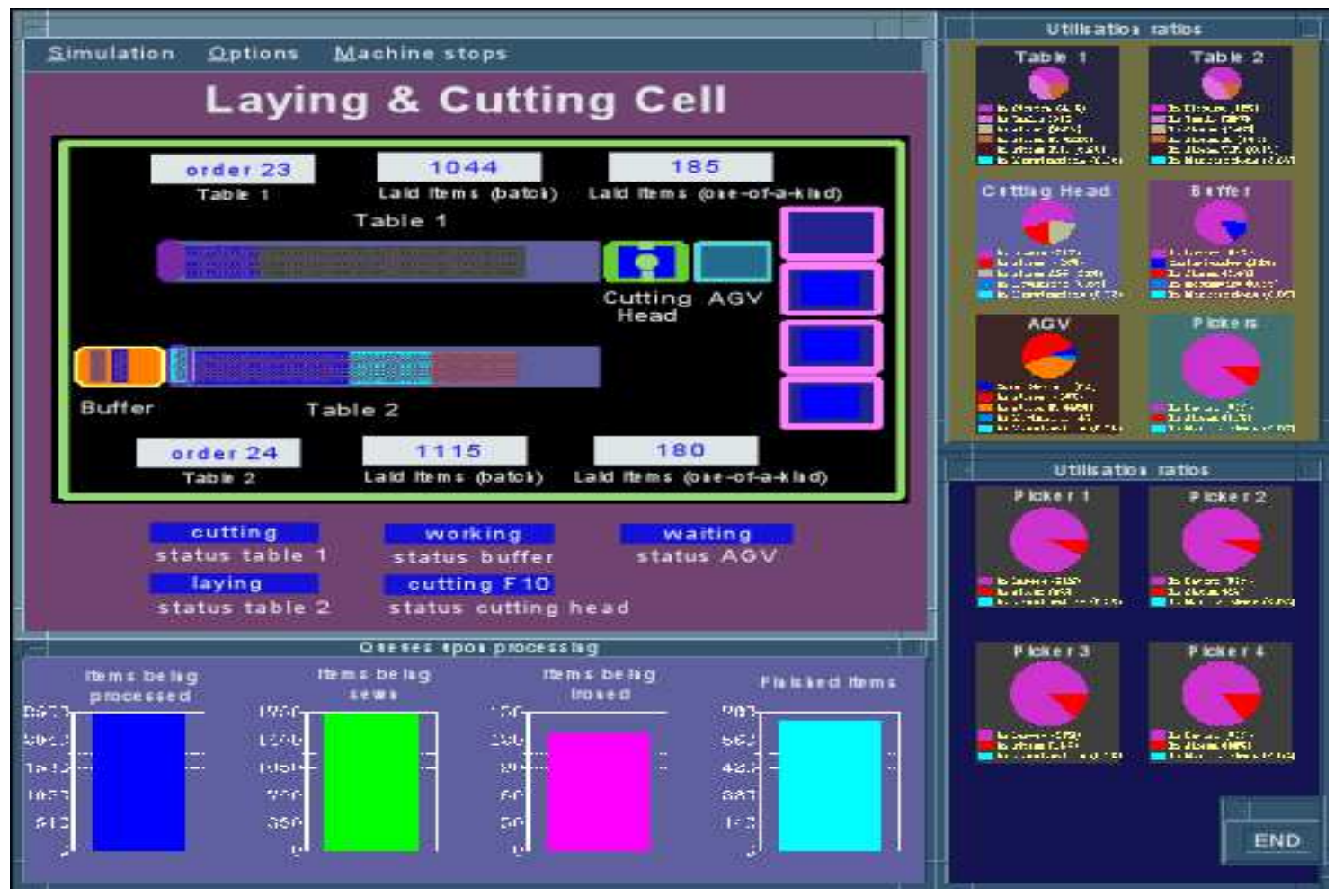

Figure 12. Example LCC-SIFIP display for the process monitoring.

This information can be exploited to permit the effective interlaced processing of:

- sorted batches, over tactical spans, with delivery to decentralised special shops;

- one-of-a-kind items, to yield quick-response issues, processed in the same shop.
Planning for combined-mode operations (batch plus one-of-a-kind) has critical middle phase at fabric cutting, where automation has wide acceptance and investment return is simply related to productivity.

The Fig. 13 gives a view of the cutting tables, with the lay-out of the piled-up pieces, ready for striping and collec- 
tion in sorted packages to be forwarded to the sewing sections. To understand the actual effectiveness of the on-progress fabrication agenda, the display provides the statistics by current and accumulated figures.

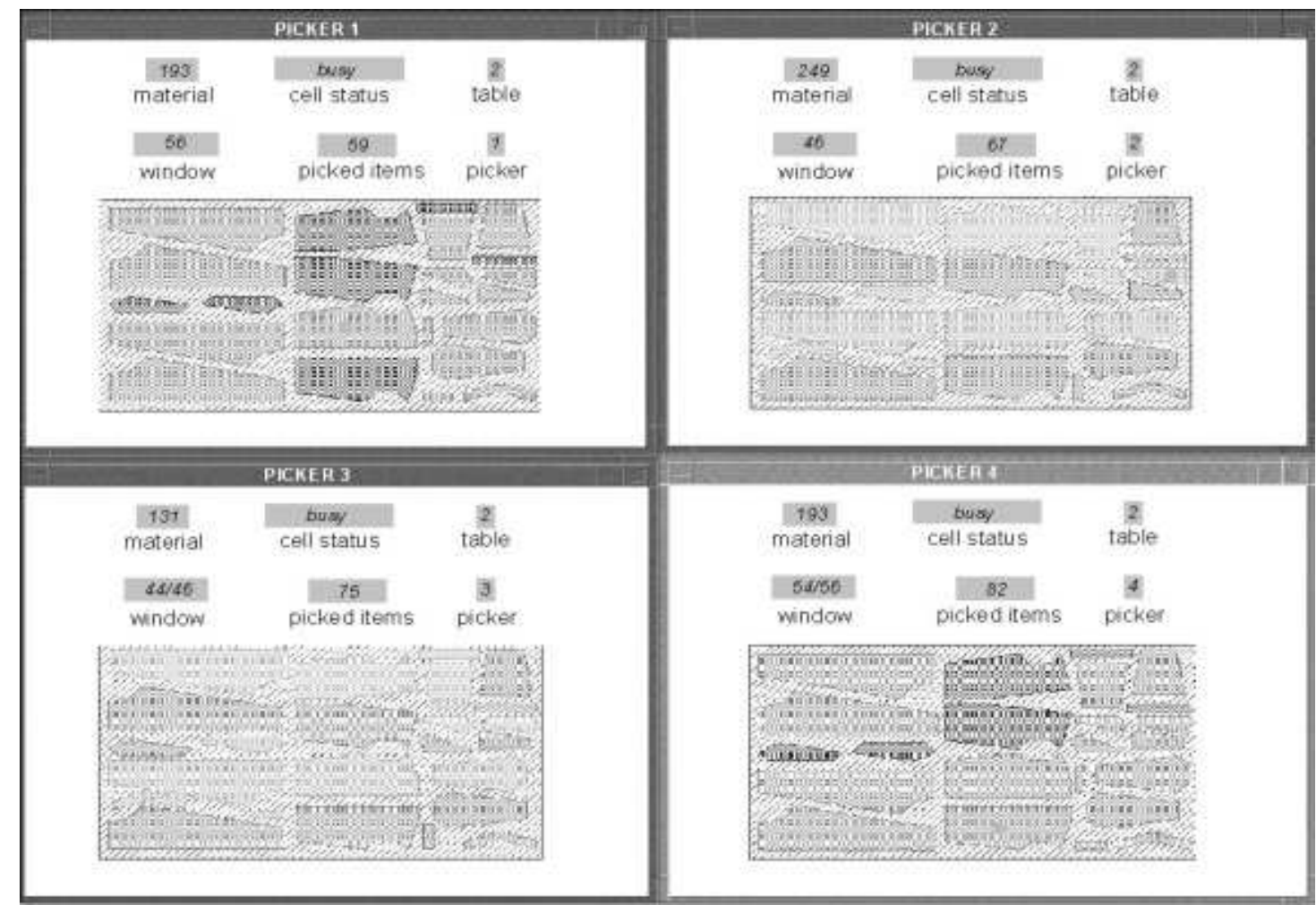

Figure 13. Characterising output arrangement of the cutting units.

The 〈intelligent setting addresses possible benefits e.g., Fig. 14:

at the organisational range: to select product mix variability under process-attuned managers;

- at the coordination range: to optimise production agendas, under decentralised controllers;

- at the operational range: to deal with on-process discontinuities, under real-time supervisors.

(a)

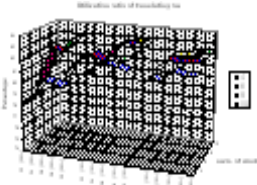

(b)

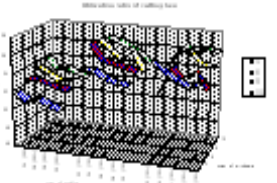

(c)

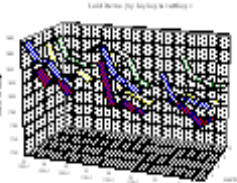

\section{.}

(d)

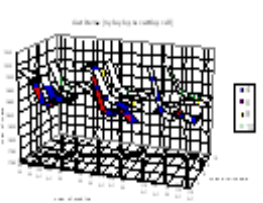

Figure 14. Example work-loads of: (a) buffer, (b) cutting head, (c) laid spans, (d) cut items.

The Fig. 15 shows the overall cell productivity in terms of garments ready for sewing per hour. The productivity of the layout and cutting cell depends on several factors. Fig. 14 and Fig. 15 indicate dependence on current batch size, number of reserved roll places for the feeding buffer (cradles) and also on the number of extra layout windows, added to the regular format. Crafty expedients (on-line resort to fabric defect maps, combined-mode batch/single processing, etc.) are requisites of intelligent manufacture; benefits depend on a large number of cross-related facts, conditioned by material and data flows, whose efficiency critically depends on automatic processing [14].

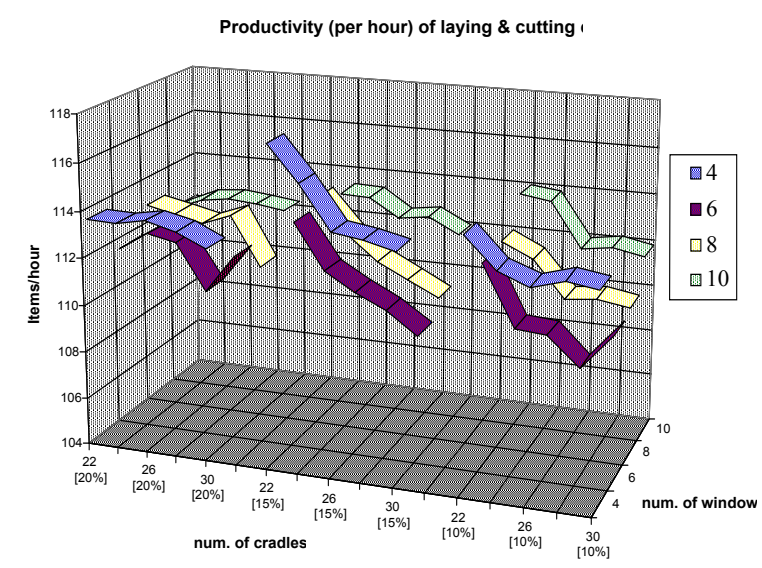

Figure 15. Cutting cell productivity for different setups.

\section{Sewing Section Mixing}

The sewing of garments is critical step in the clothing manufacture. The stage tends to be most routine and labour 
intensive and is responsible for the productive break-up out of the developed countries. The sewing section (for already assorted pieces) may be considered for operations at decentralised locations, where the lowest competitive labour rates are available. On the other hand, the sewing process is a prime candidate for intelligence sharing and it is especially desirable to operate it locally, for the manufacture of customised garments, when quality data are specifically addressed. Since long-term business success is critical goal, balancing added value and cost reduction against decentralisation and preserving high standing products under direct local control (e.g. by sewing supervision and adaptive scheduling) need to be considered as important issues.

To that purpose, the organisational requirements are reviewed, with aims at methods best suited for establishing and assessing improvements. The attention on virtual testing is deepened for, mainly, two reasons: the on-line request of manual operations needs focus on uncertainty and heuristics, further to the automation sharpness; the on-process decision cycle requests real-time govern modules operating with intensive information. A multiple procedure, Fig. 16, is useful preparation of the scheduled facilities.

\# facility behoviourol model: relational description of the resources dynamics and of
the decisions manifold, actually specifying the real plant evolution.
\# facility performonce evoluotion: factual metrics, measuring the return on invest-
ment by referring to varying product mixes and actual production plans.
\# facility simulotion softwore: computer-aids supplying virtual reality miming of
competing resource lay-outs and work-programs.
\# facility expert governor: integrated control-and-management units, enabling
adaptive schedules depending on the work-in-progress mixes.

Figure 16. Multiple step procedure to appraise flexibility effects in manufacture.

Customised high quality products are less affected by market saturation, but must reach interested buyers with right price and timing. Workplace organisation shall aim at distributed commitment and cooperative rewards. Data management systems require ready access to economical (finance, accounting), technical (order processing) and operation (master plan) aspects of the sales policy (item, quality, price, due-date, etc.). The evolution of this process is related to the concurrence of material and data flow, with changes in both driven by the control flow. Economy of scope enables continuous adaptation of current tasks and decision support, tuned to business achievements. Clearly, this upgrading rationale stems from the ability to quantitatively assess the on-going process and issues of the considered changes.

In such frame, the SCX-SIFIP package is built incorporating G2 language features, to fulfil on-process interfacing through rule-based govern modules with real-time ability, covering a wide set of production scenarios, by rule-invoking abilities, Fig. 17.

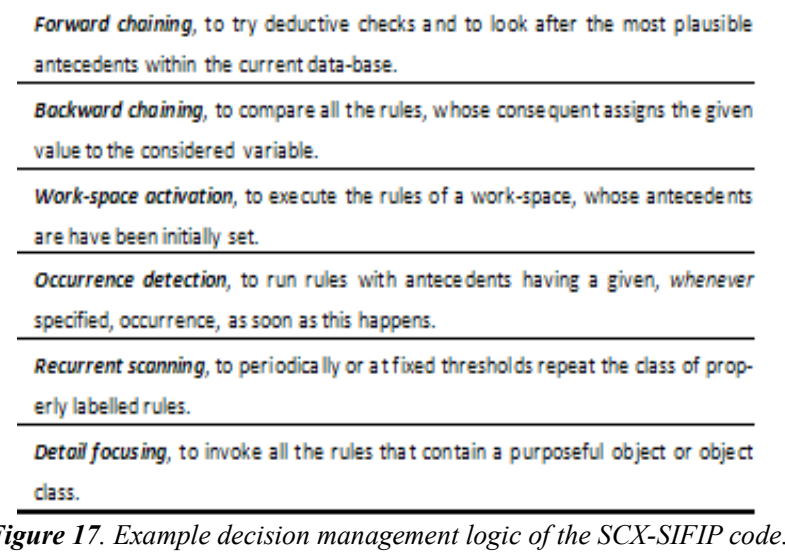

A case application is developed, exemplifying the expert simulation potentials. The resources of a standard processing cell include the: - set of sewing heads; - I/O buffer; • carrying pallets; - production batches; - product mixes; • in-process operator; $\bullet$ supplying station (laying/cutting section); • delivery station (finishing section); $\bullet$ dispatching service; • intermediate stores; • quality control set-up. A few comments recall only on the main functional prerequisites. Each cell is fed by a shuttle (on a rail) and possesses a buffer. An automatic loading/unloading rig positions the individual pallets. The operator, placed in front of the (turn by turn) pre-set sewing head, is charged to handle the pieces to be joined. The sewing and quality modules acknowledge these and data are displayed, so that the operator follows standard schedules, Fig. 18, with a continuous check on the on-going operations. The duty list needs be completed in every detail, changing the location of the sewing head, with the specified elemental tasks.

Arrival of shuttle and buffer input loading, with the pallet carrying the assorted
parts.
Acknowledgement of work schedules.
Cycle consistency and transfer of the given pallet to the buffer.
Check of the sewing heads correct setting (or reprogramming at a new product mix).
(Manual) pieces handling and positioning (with the help of special fuxtures).
Junction of the pieces by (automatic) sewing operations and items' putting back.
Handling.
Forwarding the pallet to the next work.
Station and repeating the job as programmed.
Loading the buffer output with the finished items pallet (and request of new shut-
tles).
Removal of the pallet (dispatching policy can be tuned to deal with a set of pallets).

Figure 18. Typical operation sequence of robot assisted sewing cells.

The existing sewing heads possess several pre-set options. Simplest devices need only be set for differential or upper fabric transport and for choosing: stitch length, foot pressure, seam speed and thread tension. A cell station (and operator) is actually enabled only for the given on-going production, Fig. 19. The sewing heads for normal stitching are, thus, 
occasionally moved in or out, depending on product type and duty list. Pallet buffering offers a critical benefit, mainly, when a varying product mix is processed and the delivery policy can be optimised to reduce the head reprogramming.

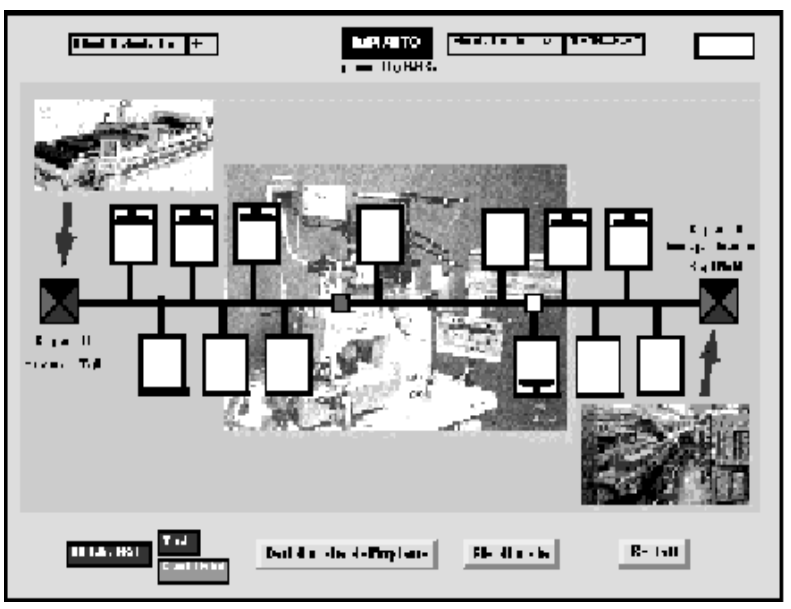

Figure 19. Example robot assisted sewing section layout.

The operator is a critical element; the simulation model needs to deal with random (human-like) job execution times and unexpected stops. The section logistics provide effective means to face flexibility on condition of having in-between stores and to supply selective dispatching. Pallet operations depend on delivery resources (carrying shuttles and loading/unloading rigs) and on shop information system (a message passing mechanism to specify the work-in-progress, etc.). An example study for the underwear manufacturing considers the assembly as composed of three parts by eight seams and finished with waist and leg elastic bands. Cost analysis provides the basic data to weigh the cycle effectiveness. Fixed and variable costs are detailed, including cell set-up, workstation outfitting, sewing heads, local buffering, loading/unloading rig, pallets, shuttles, track layout, etc.; these entities typify by unit investments: operator and supervisor attendance, power supply and maintenance of stations, transporters, etc., overheads and general charges, etc. and also the operating fees must be accurately specified.

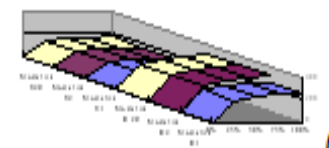

(a)

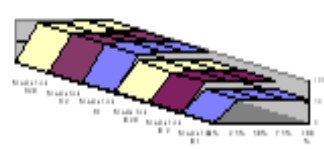

(b)

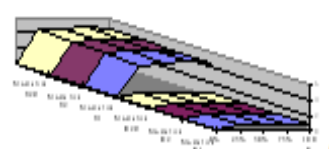

(c)

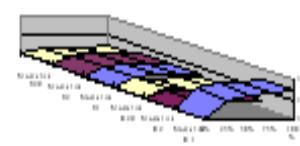

(d)

Figure 20. Slow AGV deeds: (a) throughput, (b) utilisation ratio, (c/d) in/out average queue.

Without going too far into details, basic assessments permit comparing productivity measures, observed as average figures over one or more work-shifts, modifying the assembly cycles, front-end sewing units and section logistics.
Duplication of sewing heads requires extra supply paths, provided by two shuttles sharing the same track; buffered lay-outs are safer for flexibility issues. The plots of Fig. 20 and Fig. 21 show the cell throughputs, when one or two sewing units are allocated and different shuttle speeds $(0.5$ or $1.0 \mathrm{~m} / \mathrm{s})$.

The flow balancing is better achieved at the lower speed (unless part assorted picking has a prior processing). The results consider this dispatch policy, looking at cell throughput, workstation utilisation ratio and input or output, buffering capabilities.
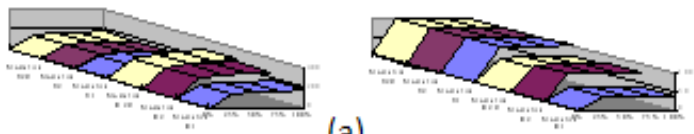

(a)
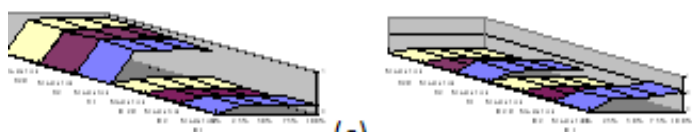

(c)

Figure 21. Fast AGV deeds: (a) throughput, (b) utilisation ratio, (c/d) in/out average queue.

Compared to the previous case examples, the present distributed information system needs explicitly to deal with workers specialisation and adeptness, included in the duty cycles as front-end operators. In this respect, the shop-floor software requires deeper concern, to make easy the interfacing with low structured environments (and not with thoroughly automatic fixtures) and to support unsteady duty cycled and heuristic decision keeping modes as inherent feature at work-cycles level.

\section{Concluding Remarks}

The intensive use of robotics and intelligent manufacture is important in competing successfully in today's international economy. The survey considers the use of these tools within the clothing industry, where low cost labour has especially driven the marketplace, in recent decades, toward productive break-up, with drawbacks on preserving the process control uniformity. Several hints are described for the use of modern manufacturing tools in the production flow and management of the related operations [15]. Emphasis is placed on aiming at high quality product in a customised production environment. The analysis begins by exploring automated retrieval of fabric from warehouse; it continues with the laying and cutting process, using combined mode schedules, mixing batch and one-of-a-kind delivery; it concludes by considering the assembly and sewing aspects of the production process. For the clothing manufacturers of developed countries, the automation concepts presented here offer an alternative (and perhaps the only alternative) to out-sourcing and overseas production.

Intelligent manufacturing is option well established, showing the new ideas of the economy of scope after centuries of economy of scale as winning reference for the in- 
dustrial enterprises. The option is linked with factory automation concepts, thus with the idea of removing front-end workers from the material flow lines by an extended use of robots or similar rigs. The approach is characterising feature of industrialised countries, somehow connected to the availability of high-tech contrivances, with market-driven production at buyers' satisfaction and with social requests for workers' better life-conditions. The clothing businesses have taken very little part to the evolution. The facts appear puzzling, at least, if one ponders the market-share of the textile and garment production, the value creation role of the fashion segments and the benefits of direct and strong links between users and supply-chain.

On these premises, the study looks after a structured approach to intelligent manufacturing, based on an integrated management of the information flow, while the material one progresses. The effectiveness depends on exploiting flexibility, to reach return on investment by market-driven production plans, timely adapted to grant buyers' satisfaction. This goal requires time-varying schedules, ruled by on-process govern decision schemata, continuously up-dated by a company-wide information system. The criticality, more than on the factory automation, outcomes from the process visibility and knowledge share. To that purpose, the shop data flow instrumentation and the manufacture processes simulation software are standard requirements. The latter makes possible to assess the effects of flexibility on the different (strategic, tactical or execution) ranges, by tests accomplished in virtual reality, according to techniques properly discussed in the paper.

The all matter is tackled with concern of practical requests and fallouts. The general set-ups are, in principle, properly defined, since they need correspond to the merging into the material flow of the all worthy data, so that individual work-tasks are timely modified to grant the business achievements, completely exploiting the available resources and reliably propagating the forecast quality [16]. The practical implementation of these ideas leaves, however, open points, particularly at the instrumental front-end, when investments in high-tech hardware are weighed against improvements in effectiveness. Certainly, the solutions are not unique; every issue is a compromise between competing requests and these are made efficient, under specified contexts. Then, for explanatory purposes, example applications are considered, covering typical sections of the garments manufacturing cycle and addressing problems of three different contexts.

The first context deals with the fabric storing and sorting sections. In front of large and time-varying product mixes, the efficiency of these sections becomes increasingly relevant; the case development aims at the integrated management of cloth data (included each bolt failure maps), so that the work-plans setting might progress according to optimal schedules, without surprises of defective parts to be replaced. The development is made possible after a thorough-out re-design of fabric warehousing, with extension of the automation level, in order that all pertinent data on cloth quality is easily monitored, shared, exploited and up-dated. The second one addresses the laying and cutting sections, already fully operated by unmanned mode. The upgrading, here, looks at knowledge intensive setting, for the on-process management of discontinuities, making easy the combined-mode (batch and one-of-a-kind) schedules to expand the plant flexibility and the early compensation of defective parts (using fabric fault maps). The development specifically requires re-thinking the enterprise policy, with minor changes of the computer interfaces, but proper resetting of the shop information system. The third context highly differs, as we refer to assembly and sewing sections, still mainly operated by front-end workers. It should be said that the setting of fully automatic sewing cells has been conceived and actual fixtures exist for the mass production of steady specialised articles; this is possibly not consistent with economy of scope and a different implementation is outlined, with front-end workers and shared information steering duty-cycle changes. Again, proper improvements are obtained, with benefits not requesting investments in hardware, rather the work-plans up-dating depending on the process progression.

The extended use of distributed intelligence and the techniques of scope economy are important factors in competing successfully in today's world. This survey considers the use of these tools within the clothing industry, where low cost labour has especially driven the marketplace, in recent decades, toward productive break-up, with relevant drawbacks on preserving the process control uniformity. Several hints are described for the use of modern manufacturing tools in the production flow of clothing and in the management of the related operations. Emphasis is placed on maintaining a high quality product in a customised production environment. The three example applications distinguish each other for front-end automation; the existence of such highly different levels characterises clothes industry, and return on investment cannot justify large hardware innovation. The situation drastically changes when the shop information systems are considered: relevant benefits follow from process visibility and on-process adaptation, making possible economy of scope, as the example cases show.

\section{References}

[1] M. Acar, 1995: "Intelligent textile machines and systems", M. Acar, ed.: 'Mechatronics Design in Textile Engineering', Kluver Acad. Press, pp. 61-66.

[2] N. A. Hunter, R. E. King, H. L. W. Nuttle, J. R. Wilson, 1993: "North Carolina apparel pipeline modelling project", Intl. J. of Clothing Science and Technology, 5, pp. 19-24.

[3] D.H. Kincade, 1995: "Quick response management system for the apparel industry: definition through technologies", Clothing and Textiles Research J., 13 (4), pp. 245-251, UK.

[4] U. Meyer, 1994: "Automation and control in textile production", Globalisation: Technological, Economic and Environmental Imperatives - 75th World conference of Textile 
Institute.

[5] A. Seyam, F. Sun, 1994: "Manufacturing technology for apparel automation: lay-up module, part II", Intl. J. of Clothing Science and Technology, 6 (1), pp. 5-3, MCB University Press.

[6] R.C. Michelini, G.M. Acaccia, M. Callegari, R.M. Molfino, R.P. Razzoli, 1997: "Shop controller-and-manager for intelligent manufacturing", S. Tzafestas, Editor: Management and Control of Manufacturing Systems, Springer Verlag, London, pp. 219-254.

[7] C. Antonelli, 2008: "The economics of innovation: critical concepts in economics", Rutledge, New York.

[8] G.L. Kovàcs, P. Bertòk, G. Heidegger, Eds., 2002: "Digital enterprise challenge: lifecycle approach to management and production", Kluwer Acad. Pub., Boston.

[9] J.X. Liu., Ed., 2005: "New developments in robotics research", Nova Sci. Pub., New York.

[10] R.C. Michelini, 2009: "Robot age knowledge changeover",
Nova Sci. Pub., New York.

[11] J. Olhagher, F. Persson, Eds., 2007, Advances in production management systems, Springer, Boston.

[12] G.J. Olling, G. Jacucci, K. Preiss, M. Wozny, Eds., 1998: "Globalisation of manufacturing in the digital communication era: innovation, agility and the virtual enterprise", Kluwer Acad. Pub., Norwell.

[13] F.Y. Phillips, 2001: "Market-oriented technology management: innovating for profit in entrepreneurial time", Springer, Berlin.

[14] S. Zhang, L. Lin, A. Kumar, 2009: "Materials characterisation techniques", CRC Press, Abingdon.

[15] N.L. Russo, B. Fitzgerald, J.I. DeGross, Eds., 2001: "Re-aligning research and practice in information systems development: the social and organizational perspective, Kluwer Academic", New York.

[16] G. Taguchi, E.A. Elsayed, T.C. Hsiang, 1989: "Quality engineering in production systems", McGraw Hill, New York. 